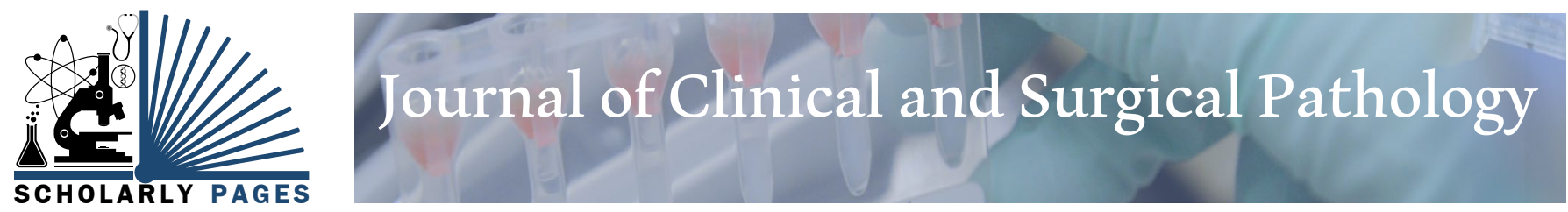

\title{
Immunoglobulin G4 Positive Plasma Cells in Chronic Liver Disease
}

\section{Claire J Detweiler', Lauren M Allen', Deepti M Reddi', Shannon J McCall', Cynthia D Guy $^{1}$, Mustafa R Bashir ${ }^{2}$, Julius M Wilder ${ }^{3,4}$ and Diana M Cardona ${ }^{1^{*}}$}

\author{
${ }^{1}$ Department of Pathology, Duke University Medical Center, Durham, NC, USA \\ ${ }^{2}$ Department of Radiology, Duke University Medical Center, Durham, NC, USA \\ ${ }^{3}$ Department of Medicine, Duke University Medical Center, Durham, NC, USA \\ ${ }^{4}$ Duke Clinical Research Institute, Duke University Medical Center, Durham, NC, USA
}

\begin{abstract}
Introduction: IgG4-related disease was first defined in autoimmune pancreatitis but is known to affect other organs, including the biliary tree and liver. The aim of this study was to assess IgG4-positive plasma cells in a spectrum of chronic liver diseases.

Materials and methods: A retrospective search was performed for liver specimens of various clinically-confirmed chronic liver diseases. A chart review was performed. H\&E, trichrome, and IgG4 slides were reviewed. The numbers of total and IgG4-positive plasma cells were quantified. The consensus ratio of $>40 \%$ IgG4-positive:total plasma cells and $>10$ IgG4plasma cells per high power field (HPF) were the thresholds used to define a significant increase.

Results: Three IgG4-related disease, 19 primary biliary cirrhosis, 18 primary sclerosing cholangitis, 22 autoimmune hepatitis, 25 chronic hepatitis $C$, and 1 secondary sclerosing cholangitis of unknown etiology cases were identified. A significantly elevated cutoff ratio was seen in only one IgG4-related disease and one case with unknown etiology. Two chronic hepatitis $\mathrm{C}$ cases had an elevated cutoff ratio but overall low numbers of plasma cells ( $<1$ IgG4-positive plasma cells per HPF). The sensitivity and specificity of the $>40 \%$ IgG4-positive:total plasma cells ratio were $33.3 \%$ and $96.5 \%$, respectively. The sensitivity and specificity of the > 10 IgG4-positive plasma cells per HPF were both $100 \%$.
\end{abstract}

Discussion: Elevated IgG4-positive plasma cells on liver biopsy are not completely specific for IgG4-related disease and a cutoff ratio of $>40 \%$ IgG4-positive:total plasma cells has low sensitivity. Liver biopsies with a prominent plasma cell infiltrate warrant IgG4 immunohistochemistry to help evaluate IgG4-related disease; however, a lower threshold of IgG4positive plasma cells may indicate a significant increase.

Keywords

IgG4, Immunoglobulin G4, Plasma cell, IgG4 related sclerosing cholangitis

\section{Introduction}

Immunoglobulin G4-related disease (IgG4RD) is a systemic immune-mediated disease characterized by elevated serum IgG4 concentration and tissue infiltration by IgG4positive plasma cells [1-4]. Although IgG4RD was first defined in the pancreas, this entity has now been reported in nearly every organ system of the body $[2,3,5]$. Within the biliary tree and liver, IgG4RD is a known cause of sclerosing cholangitis, biliary strictures, sclerosing cholecystitis, gallbladder nodules and thickening, and inflammatory pseudotumors of the liver [4-6]. Among patients with systemic IgG4RD, 19.5\% have biliary tree or gallbladder involvement and $4 \%$ have liver involvement. IgG4-related sclerosing cholangitis is strongly associated with IgG4-related pancreatitis, with $83 \%$ of patients with

\footnotetext{
${ }^{*}$ Corresponding author: Diana M Cardona, MD, Department of Pathology, Duke University Medical Center, PO Box 3217, Durham, NC 27710, USA, Tel: +1-919-613-5208, Fax: +1-919-681-8976, E-mail: diana.cardona@duke.edu

Received: September 29, 2017; Accepted: December 26, 2017; Published online: December 28, 2017

Citation: Detweiler CJ, Allen LM, Reddi DM, et al. (2017) Immunoglobulin G4 Positive Plasma Cells in Chronic Liver Disease. J Clin Surg Pathol 1(1):1-6
} 
cholangitis also having pancreatitis [5]. Diagnostic criteria for IgG4RD include characteristic clinical manifestations, elevated serum IgG4 concentrations, and histopathologic changes such as infiltration of IgG4+ plasma cells [4,7]. The 2012 consensus guidelines for the histopathologic cutoffs for IgG4RD in any organ are an IgG4+:IgG+ plasma cell ratio $>40 \%$ and $>10$ IgG4 + plasma cells per high power field (HPF). To date, no organ specific data for IgG4-related sclerosing cholangitis exists [8]. However, histologic features suggestive of IgG4-related sclerosing disease include a dense lymphoplasmacytic infiltrate, storiform fibrosis, and obliterative phlebitis [4]. There are numerous published studies on the topic of IgG4 response in primary sclerosing cholangitis (PSC), but less so in other autoimmune related liver diseases such as primary biliary cirrhosis (PBC) and autoimmune hepatitis (AIH) [9-13]. Also, these studies did not apply the 2012 cutoff guidelines for the diagnosis of IgG4RD. The aim of this study was to assess the presence of IgG4+ plasma cells in a spectrum of chronic liver diseases utilizing the 2012 diagnostic guidelines for IgG4RD.

\section{Materials and Methods}

A retrospective search of the Duke University Hospi- tal (Durham, NC) laboratory information system from January 1, 2000 to January 31, 2007 was performed for cases of clinically confirmed $\mathrm{PBC}$ and $\mathrm{AIH}$ as well as up to 25 cases of clinically confirmed chronic hepatitis $\mathrm{C}$ (HCV). Additionally, a retrospective search from January 1, 2000 to June 30, 2015 for cases of clinically confirmed PSC was performed. All except one of the cases of PSC were diagnosed clinically and confirmed with radiologic imaging. The exception is one case for which the underlying clinical etiology of liver disease remained unknown but the diagnosis of PSC was suspected on liver histopathology. Additionally, a retrospective search from January 1, 2004 to June 30, 2016 for cases of clinically confirmed IgG4-related sclerosing cholangitis was performed. During this search a biopsy with pathology results concerning for IgG4RD in a patient with cholangiocarcinoma was identified and omitted from this study.

A chart review was performed, and relevant clinical information and laboratory data were recorded. Liver function tests (LFTs) and immune serologies, including serum IgG4 levels, were also recorded when applicable. The reference range for serum IgG4 level was 2.4 to 121.0 $\mathrm{mg} / \mathrm{dL}$. Liver biopsy specimens were fixed in $10 \%$ neu-

Table 1: Demographic and histopathologic features of all needle core biopsies.

\begin{tabular}{|c|c|c|c|c|c|c|}
\hline & IgG4RD & PBC & PSC & AlH & HCV & $\begin{array}{l}\text { Unknown } \\
\text { etiology }\end{array}$ \\
\hline Total no. of cases & 3 & 19 & 18 & 22 & 25 & 1 \\
\hline Age at biopsy (years, avg/range) & $51 / 32-62$ & $53 / 30-74$ & $36 / 11-61$ & $43 / 7-73$ & $48 / 23-67$ & 53 \\
\hline Male:Female & $2: 1$ & $1: 18$ & $7: 11$ & $9: 13$ & $14: 11$ & $1: 0$ \\
\hline $\begin{array}{l}\text { No. positive ANA titer cases/no. } \\
\text { cases performed }^{1}\end{array}$ & $1 / 1(100 \%)$ & $11 / 16(69 \%)$ & $9 / 14(64 \%)$ & $21 / 22(95 \%)$ & $3 / 3(100 \%)$ & N/A \\
\hline $\begin{array}{l}\text { No. positive ASMA titer cases/no. } \\
\text { cases performed }{ }^{1}\end{array}$ & $0 / 1(0 \%)$ & $0 / 10(0 \%)$ & $0 / 10(0 \%)$ & $17 / 19(89 \%)$ & $0 / 2(0 \%)$ & $0 / 1(0 \%)$ \\
\hline $\begin{array}{l}\text { No. positive AMA titer cases/no. } \\
\text { cases performed }^{1}\end{array}$ & $\mathrm{~N} / \mathrm{A}$ & $17 / 19(89 \%)$ & $0 / 6(0 \%)$ & $1 / 13(8 \%)$ & $0 / 1(0 \%)$ & $0 / 1(0 \%)$ \\
\hline AST (avg; U/L) & 136.7 & 73.3 & 112.9 & 405.5 & 49.8 & 80.0 \\
\hline ALT (avg; U/L) & 125.0 & 95.2 & 144.8 & 457.5 & 60.2 & 62.0 \\
\hline Alkaline phosphatase (avg; U/L) & 516.0 & 258.4 & 519.3 & 189.0 & 89.6 & 143.0 \\
\hline Total bilirubin (avg; mg/dL) & 3.9 & 0.8 & 1.5 & 4.7 & 0.5 & 6.0 \\
\hline $\begin{array}{l}\text { Serum IgG concentration (avg/ } \\
\text { range) })^{2}\end{array}$ & $2587 / 1420-4730$ & $1256 / 1030-1510$ & $1432 / 845-2360$ & $3323 / 1240-6060$ & N/A & N/A \\
\hline $\begin{array}{l}\text { Serum IgG4 concentration (avg/ } \\
\text { range) })^{3}\end{array}$ & $65 / 14.2-125$ & N/A & N/A & N/A & N/A & N/A \\
\hline Clinical history of pancreatitis & $1(33 \%)$ & $N / A$ & $N / A$ & N/A & N/A & N/A \\
\hline Plasma cells/5 HPF (avg/range) & 269/140-376 & $73 / 5-289$ & $10 / 0-38$ & $131 / 19-345$ & $16 / 2-44$ & 48 \\
\hline $\begin{array}{l}\text { lgG4+ plasma cells/5 HPF (avg/ } \\
\text { range) }\end{array}$ & $101 / 80-127$ & $0.3 / 0-2$ & $0.4 / 0-6$ & $8 / 0-29$ & $0.5 / 0-3$ & 24 \\
\hline $\begin{array}{l}\text { lgG4+ plasma cells:total plasma } \\
\text { cells }(\%)\end{array}$ & $43 \% / 27-69 \%$ & $1 \% / 0-7 \%$ & $0.2 \% / 0-16 \%$ & $5 \% / 0-16 \%$ & $6 \% / 0-50 \%$ & $52 \%$ \\
\hline Eosinophils/5 HPF (avg/range) & $51 / 7-130$ & $23 / 5-120$ & $3 / 0-11$ & $13 / 1-29$ & $12 / 1-28$ & 55 \\
\hline
\end{tabular}

${ }^{1}$ ANA, AMA, AMSA considered positive if titer $\geq 1: 160 ;{ }^{2}$ Reference range of serum IgG concentration $588-1573 \mathrm{mg} / \mathrm{dL} ;{ }^{3} \mathrm{Reference}$ range of serum IgG4 concentration $2.4-121.0 \mathrm{mg} / \mathrm{dL}$.

AlH: Autoimmune Hepatitis; ALT: Alanine Transaminase; AMA: Anti-Mitochondrial Antibody; ANA: Anti-Nuclear Antibody; ASMA: Anti-Smooth Muscle Antibody; AST: Aspartate Transaminase; GGT: Gamma-Glutamyl Transferase; HCV: Chronic Hepatitis C; HPF: High Power Field; IgG: Immunoglobulin G; PBC: Primary Biliary Cirrhosis; PSC: Primary Sclerosing Cholangitis. 
tral buffered formalin, paraffin embedded, and 5-micron thick sections were taken. Immunohistochemistry (IHC) using monoclonal IgG4 antibody (Life Technologies/Invitrogen, Cell Marque MRQ-44, 1:200) was performed on all of the collected cases. Hematoxylin \& eosin (H\&E), trichrome, and IgG4 IHC slides were reviewed by two pathologists, blind to the clinical information. The features of the inflammatory infiltrate for each biopsy were recorded including the nature, location, and intensity of the inflammation as well as the stage of fibrosis and the presence or absence of storiform fibrosis. The presence or absence of venulitis and obliterative phlebitis were also recorded. In the areas of highest cellularity, the number of plasma cells, IgG4+ plasma cells, and eosinophils were counted in 5 HPFs. For this study, a HPF was defined as a $40 \mathrm{X}$ field (field of view $0.55 \mathrm{~mm}$ ). A cutoff ratio of $>$ 40\% IgG4+:total plasma cell ratio and > 10 IgG4+ plasma cells per HPF were used to define a significant increase in IgG4+ plasma cells, similar to the 2012 consensus guidelines for the histopathologic cutoffs for IgG4RD in any organ of $>40 \%$ IgG4+:IgG+ plasma cells [8]. Each of the core biopsies was also graded by the pathologists: $\mathrm{HCV}$ and AIH cases were graded and staged using the Batts and Ludwig scale [14]. PSC cases were graded and staged using the Ishak scale for PSC [15]. PBC cases were graded and staged using the Ludwig scale for PBC [16].

Table 2: Histopathologic features of needle core biopsies with an elevated $\lg \mathrm{G}+$ :total plasma cells ratio ${ }^{1}$.

\begin{tabular}{|l|l|l|l|l|}
\hline Clinical diagnosis & IgG4RD & $\begin{array}{l}\text { Unknown } \\
\text { etiology }\end{array}$ & HCV & HPV \\
\hline $\begin{array}{l}\text { Plasma cells/5 HPF } \\
\text { (average) }\end{array}$ & 140 & 48 & 2 & 7 \\
\hline $\begin{array}{l}\text { IgG4+ plasma cells/5 HPF } \\
\text { (average) }\end{array}$ & 97 & 25 & 1 & 3 \\
\hline $\begin{array}{l}\text { IgG4+ plasma cells:total } \\
\text { plasma cells }\end{array}$ & $69 \%$ & $52 \%$ & $50 \%$ & $43 \%$ \\
\hline Eosinophils/5 HPF (average) & 7 & 55 & 14 & 19 \\
\hline
\end{tabular}

${ }^{1}$ Significant increase was defined as $\geq 40 \%$. HCV: Chronic Hepatitis C; HPF: High Power Field; IgG: Immunoglobulin G; IgG4RD; Immunoglobulin G4-related disease.

\section{Results}

Needle core biopsies were available for 3 IgG4RD, 19 PBC, $22 \mathrm{AIH}, 18 \mathrm{PSC}, 25 \mathrm{HCV}$, and 1 secondary sclerosing cholangitis of unknown etiology cases (Table 1). A significantly elevated (>40\%) cutoff ratio was seen in 1 IgG4RD, 1 secondary sclerosing cholangitis of unknown etiology, and $2 \mathrm{HCV}$ cases (Table 2). A significantly elevated $(>10)$ number of IgG4+ plasma cells per HPF was only seen in the $3 \operatorname{IgG} 4 \mathrm{RD}$ cases. Only one IgG4RD case was equivocal for storiform fibrosis with all other cases negative and there were no cases that demonstrated obliterative phlebitis.

Out of 3 IgG4RD cases, one (33\%) case had an IgG4+:total plasma cell ratio of $69 \%$ with 28.0 plasma cells per HPF and 19.4 IgG4+ plasma cells per HPF (Figure 1). The liver biopsy also demonstrated abundant secretion of IgG4 into the surrounding soft tissues. Chart review revealed a 53-year-old male with a history of cirrhosis initially diagnosed as PSC. Serum IgG4 levels were not elevated at the time of his liver biopsy $(14.2 \mathrm{mg} / \mathrm{dL})$. A year prior to his liver biopsy the patient had bone marrow and lymph node biopsies performed which showed polyclonal plasma cell infiltrates with IgG4 staining seen in the bone marrow (significant, but limited by high background staining) and lymph node (nearly 100\% IgG4+:IgG+ ratio in plasma cells). At one point he was treated with high dose steroids for thrombocytopenia, which resulted in significant improvement in his LFTs. Upon future follow up 4 years after the initial liver biopsy, the patient was overall stable with his chronic liver disease aside from some jaundice after a surgical procedure and was maintained on low dose prednisone for his IgG4RD.

The remaining two IgG4RD cases both had significant numbers (> 10 per HPF) of IgG4+ plasma cells (16.0 and 25.4 per HPF) but with ratios less than the cutoff $(27 \%$ and $34 \%)$, likely due to the high numbers of total plasma cells

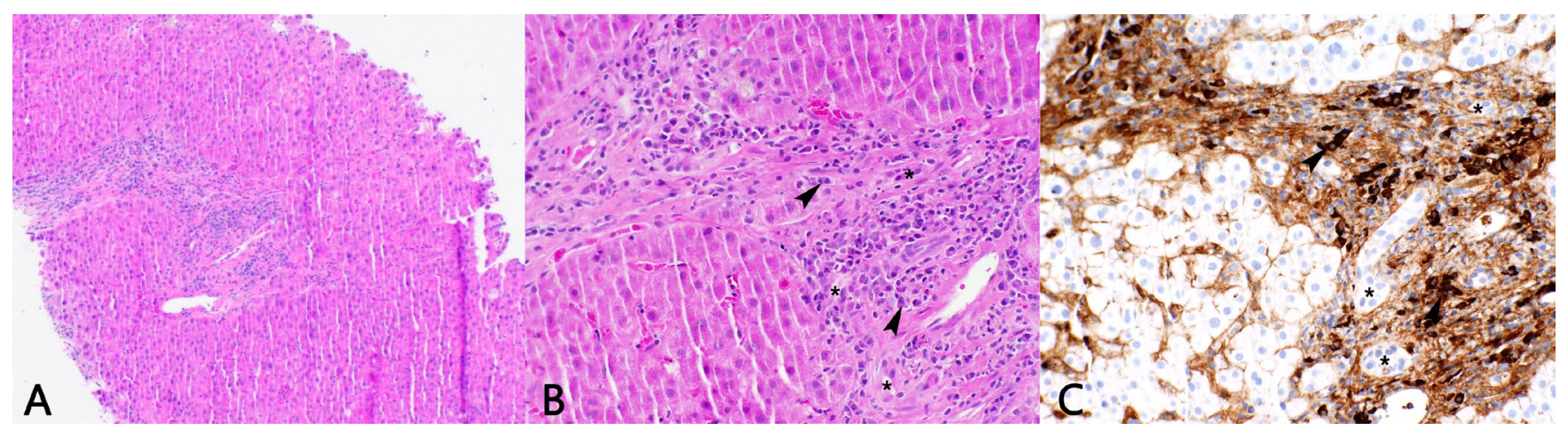

Figure 1: Photomicrographs of IgG4-related sclerosing cholangitis case with $69 \%$ lgG4+:total plasma cells demonstrating plasma cell rich infiltrate with clusters of IgG4+ plasma cells (arrows) and involved bile ducts (asterisks) with abundant secretion of IgG4 into the surrounding soft tissues (A: H\&E, 4x; B: H\&E, 20x; C: IgG4 IHC, 20x). 


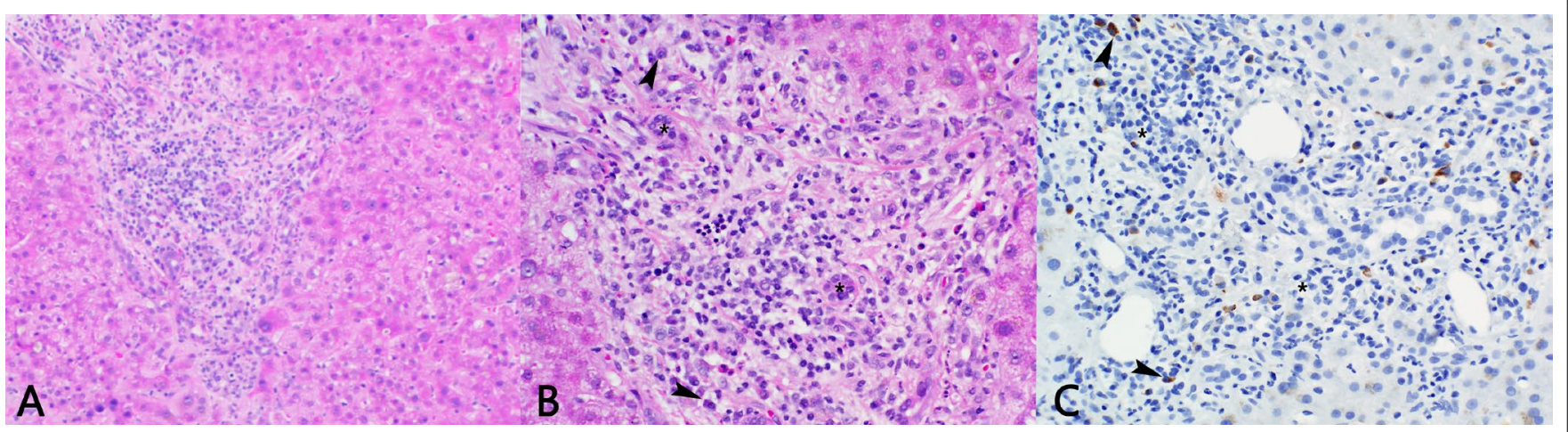

Figure 2: Photomicrographs of secondary sclerosing cholangitis of unknown etiology case with $52 \%$ lgG4+:total plasma cells showing scattered IgG4+ plasma cells (arrows) and involved bile ducts (asterisks) (A: H\&E, 4x; B: H\&E, 20x; C: IgG4 IHC, 20x).

(58.4 and 75.2 per HPF). Chart review of the first case (ratio of $27 \%$ ) revealed a 62 -year-old male with cholestatic liver disease which significantly improved with steroid therapy, which with his histology findings, was consistent with IgG4RD. Additionally, his serum IgG4 levels were mildly elevated at $125 \mathrm{mg} / \mathrm{dL}$ (reference range $2.4-121.0 \mathrm{mg} / \mathrm{dL}$ ). At his last follow up 4 years after the initial biopsy he was doing well on intermittent steroid therapy. The second IgG4RD case (ratio of 34\%) was a 32-year-old female with a history of elevated LFTs, recurrent pancreatitis, and abnormal biliary tree changes on CT scan. She underwent a partial hepatectomy for intrahepatic stones and biliary obstruction. Serum IgG4 levels were not elevated ( $54.3 \mathrm{mg} / \mathrm{dL})$. Unfortunately, the patient was lost to follow up soon after her surgery.

One case which was ultimately classified as secondary sclerosing cholangitis of unknown etiology had an elevated ratio of $52 \%$ with 9.6 plasma cells per HPF and 5.0 IgG4+ plasma cells per HPF, thus reaching the ratio threshold (> $40 \%$ ) but not the total IgG4+ plasma cell threshold ( $>10$ per HPF) (Figure 2). This case was a 53-year-old male originally diagnosed with PSC presenting with jaundice and biliary strictures on imaging. Clinical follow up 13 years after the initial liver biopsy the patient had symptom and imaging abnormality resolution without steroid treatment, bringing into doubt the initial diagnosis of PSC.

Two out of 25 (8\%) HCV cases had an elevated ratio: $50 \%$ with 0.4 plasma cells per HPF and 0.2 IgG4+ plasma cells per HPF and $43 \%$ with 1.4 plasma cells per HPF and 0.6 IgG4+ plasma cells per HPF. While these meet the cutoff ratio threshold of $>40 \%$, they are far below the total IgG4+ plasma cell count threshold (> 10 per HPF) and the elevated ratio can be attributed to the overall low number of total plasma cells. None of the PSC, PBC, or AIH cases had an elevated IgG4+:total plasma cell ratio.

\section{Discussion}

IgG4-related sclerosing cholangitis is a type of secondary sclerosing cholangitis associated with autoimmune pancreatitis $[2,5,6,9,17]$. The major clinical differ- ential diagnoses of IgG4RD in the biliary tree and liver are PSC, pancreatic cancer, cholangiocarcinoma, and hepatocellular carcinoma [2]. While malignant neoplastic processes do not typically mimic IgG4RD on histology, inflammatory processes can as both are characterized by a mononuclear inflammatory infiltrate, including plasma cells. The 2012 consensus guidelines for the histopathologic cutoffs for IgG4RD in any organ are an IgG4+:IgG+ plasma cell ratio $>40 \%$ and $>10$ IgG4+ plasma cells per HPF [8]. Although there is no organ specific data in the liver regarding IgG4RD, we used comparable cutoffs when evaluating our study cases (> 40\% IgG4+:total plasma cell ratio and > 10 IgG4+ plasma cells per HPF). A limitation of these thresholds is that liver specimens are complicated by the presence of other chronic inflammatory liver disorders, thus the aim of our study was to assess the presence of IgG4+ plasma cells in a spectrum of chronic liver diseases utilizing the 2012 diagnostic guidelines for IgG4RD.

In our study, we gathered cases of IgG4RD, PSC, $\mathrm{PBC}$, and $\mathrm{AIH}$ as representative autoimmune related chronic liver diseases. Incidentally, a case of PSC was recategorized as secondary sclerosing cholangitis of unknown etiology after chart review. The non-autoimmune control of HCV cases were selected to demonstrate that an increase in IgG4+ plasma cells present was not simply due to a non-specific inflammatory response.

Only one of the three IgG4RD cases met both the IgG4+:total plasma cell ratio (69\%) and IgG4+ plasma cells per HPF thresholds (19.4 per HPF). While the other two cases had increased IgG4+ plasma cells, they had many IgG4 negative plasma cells as well resulting in a lower IgG4+:total plasma cell ratio (27\% and $34 \%$ ). This calls into question the diagnostic sensitivity and negative predictive value of the guideline thresholds. Potentially a lower IgG4+:total plasma cell ratio, closer to $25 \%$, is significant and may indicate IgG4RD as a cause of cholangitis. Of the cases with $<40 \%$ IgG+:total plasma cell ratios, 2 of 2 (100\%) IgG4RD, 1 of 22 (5\%) AIH, 0 of 23 (0\%) HCV, 
0 of $19(0 \%)$ PBC, and 0 of 18 (0\%) PSC cases had an IgG+:total plasma cell ratio $>25 \%$. One IgG4RD case had a ratio of $27 \%$ and $16.0 \mathrm{IgG} 4+$ plasma cells per HPF. Chart review revealed a patient with cholangitis that improved with steroid therapy. The other IgG4RD case had a ratio of $34 \%$ and 25.4 IgG4+ plasma cells per HPF. This patient had cholangitis and recurrent pancreatitis, though she was lost to follow up so therapy response is unknown. We think that despite not meeting guideline thresholds for IgG4+:total plasma cells that both of these patients represent true IgG4-related sclerosing cholangitis, thus demonstrating the need for a potentially lower threshold. However, the authors recognize the overall low number of IgG4RD cases as a limitation of the study.

IgG4-related sclerosing cholangitis and PSC are indistinguishable clinically, serologically, and radiographically $[2,3,9,12]$. The most vital clinical implication of this differential diagnosis is that IgG4-related sclerosing cholangitis is responsive to steroid therapy while PSC is not $[2,6,9,18]$. Treatment options for PSC are ursodeoxycholic acid or obeticholic acid for management of cholestasis and hepatic transplantation in the setting of end stage liver disease [19]. Both present clinically as chronic cholestatic liver disease with obstructive jaundice and abnormal LFTs $[6,19]$. Both show multifocal strictures and dilatations involving both the intrahepatic and extrahepatic biliary trees on cholangiography $[2,9,19]$. In a study by Mendes, et al. 9\% of PSC cases (defined using a combination of characteristic clinical, radiographic, and histologic findings) showed an elevated serum IgG4 level, most of which did not show increased IgG4+ plasma cells on biopsy [12]. Thus, serum IgG4 concentrations do not definitively differentiate the two entities. Consequently, histopathology is a helpful tool to differentiate between IgG4-related sclerosing cholangitis and PSC as their patterns of inflammation and liver damage differ. IgG4-related sclerosing cholangitis shows transmural and portal fibrosis, storiform fibrosis, dense periportal and periluminal lymphoplasmacytic inflammation with increased IgG4+ plasma cells, and obliterative phlebitis $[2-4,6,9]$. PSC shows concentric periductal fibrosis ('onion skin' fibrosis), lymphocytic inflammation, and ductal erosion with neutrophilic inflammation [6,9]. IHC for IgG4 is another helpful tool in the differentiation of IgG4-related sclerosing cholangitis and PSC in that a highly elevated IgG4+ plasma cell level would support IgG4RD over PSC. In our study we had one case initially diagnosed as PSC with an elevated IgG4+:total plasma cell ratio (52\%) but a below threshold IgG4+ plasma cell count (5 per HPF). Upon clinical follow up 13 years after his original liver biopsy, the patient showed resolution of his symptoms and imaging abnormalities without steroid treatment. We feel these findings are not consistent with PSC nor are they classic for IgG4RD, thus we have designated it as a case of secondary sclerosing cholangitis of unknown etiology for the purpose of this study. Conversely, although untreated IgG4RD often progresses to extensive fibrosis some manifestations of the disease may remain stable over time and do not require immediate treatment [20]. This case may then represent an unusual presentation of IgG4RD without progression.

Interestingly, the AIH group had a large number of cases with IgG4+ plasma cells present compared to the other autoimmune related diseases, which only demonstrated few cases with any IgG4+ cells. However, none of the AIH cases demonstrated > 10 IgG4+ plasma cells per HPF or had a significant IgG4+:total plasma cell ratio. Thus, none of these cases met the consensus guideline cutoffs for IgG4RD. One case of AIH had below threshold but mildly increased IgG4+:total plasma cell ratio (30\%) and a below threshold but mildly increased IgG4+ plasma cell count (7.0 per HPF). Chart review revealed a 70-year-old female with jaundice and elevated LFTs on steroid therapy for systemic lupus erythematosus (SLE). Serum IgG4 levels were not tested. Based on her liver biopsy findings she was diagnosed with $\mathrm{AIH}$ and maintained on steroid therapy. 14 years after her initial liver biopsy the patient was seen at Duke Hospital again for hypothyroidism but was also noted to be in hospice care for dementia, heart failure, pulmonary fibrosis, and SLE. Her LFTs at that time were normal, though she was still taking prednisone for SLE. Approximately a year later the patient succumbed to complications of heart failure. Overall, we think that this patient likely had true AIH and not IgG4RD. This case demonstrates that lowering the IgG4+:total plasma cell ratio may decrease specificity of the histopathologic diagnosis of IgG4RD and emphasizes the need for clinico-pathologic correlation as well as evaluation of the biopsy as a whole.

We had $2 \mathrm{HCV}$ cases with IgG4+:total plasma cell ratios greater than the cutoff. However, the ratio was only significant due to overall low numbers of plasma cells and would not meet the 2012 consensus guidelines which require $>10 \mathrm{IgG} 4+$ positive plasma cells per HPF in a biopsy specimen. These cases demonstrate the importance of evaluating the total number of IgG4+ plasma cells as well as the IgG4+:total plasma cell ratio. None of the PSC or PBC cases demonstrated an elevated IgG4+:total plasma cell ratio.

Current consensus guidelines for the histopathologic diagnosis of IgG4RD are not organ specific, and it is unknown if specific criteria for liver and biliary tree involvement are needed for optimal sensitivity and specificity. While numerical cut offs can be a helpful guideline when considering IgG4RD in the differential diagnosis of a liver biopsy, it is necessary to correlate with our clinical colleagues and the overall clinical picture of 
each patient in order to make a final diagnosis. Our study showed two cases of IgG4RD that did not meet the ratio threshold but did meet the count threshold. We also had two cases of chronic HCV and one case of secondary sclerosing cholangitis of unknown etiology initially thought to be PSC that did meet the ratio threshold but not the count threshold. Consideration for lowering the ratio threshold revealed one AIH case which would have met the ratio but not count threshold. Overall, we think the consensus guideline thresholds should be used as a guide for evaluating liver biopsies for IgG4-related sclerosing cholangitis, but that each case needs to be considered individually with the clinical findings and overall biopsy findings for final diagnosis.

While a plasma cell infiltrate can be seen in a variety of inflammatory liver processes, our study demonstrates that rarely will there be a significant number of IgG4+ plasma cells. Liver biopsies with a significant plasma cell infiltrate may warrant IgG4 IHC staining to evaluate the differential diagnosis of IgG4-related sclerosing cholangitis, which is potentially responsive to immunosuppressive therapy. However, we do not recommend adhering strictly to the consensus guideline thresholds and favor individual evaluation of each case in the context of its clinical scenario and overall biopsy findings. Conversely, our data suggest IgG4 IHC within other characteristic autoimmune-like liver diseases (PBC and $\mathrm{AIH}$ ) to be of low yield.

\section{Conflicting Interests}

The authors have no conflicting interests or sources of funding to declare.

\section{References}

1. Umehara $\mathrm{H}$, Okazaki $\mathrm{K}$, Masaki $\mathrm{Y}$, et al. (2012) A nove clinical entity, IgG4-related disease (IgG4RD): General concept and details. Mod Rheumatol 22: 1-14.

2. Kamisawa T, Zen Y, Pillai S, et al. (2015) lgG4-related disease. Lancet 385: 1460-1471.

3. Mahajan VS, Mattoo H, Deshpande V, et al. (2014) IgG4-related disease. Annu Rev Pathol 9: 315-347.

4. Chen JH, Deshpande V (2017) IgG4-related disease and the Liver. Gastroenterol Clin North Am 46: 195-216.
5. Brito-Zeron P, Ramos-Casals M, Bosch X, et al. (2014) The clinical spectrum of IgG4-related disease. Autoimmun Rev 13: $1203-1210$.

6. Deshpande V (2015) IgG4-Related Disease of the Gastrointestinal Tract: A 21st Century Chameleon. Arch Pathol Lab Med 139: 742-749.

7. Ghably JG, Borthwick T, O'Neil TJ, et al. (2015) IgG4-related disease: a primer on diagnosis and management. Ann Allergy Asthma Immunol 114: 447-454.

8. Masaki Y, Kurose N, Yamamoto M, et al. (2012) Cutoff Values of Serum IgG4 and Histopathological IgG4+ Plasma Cells for Diagnosis of Patients with IgG4-Related Disease. Int J Rheumatol 2012: 580814.

9. Deshpande V, Sainani NI, Chung RT, et al. (2009) IgG4-associated cholangitis: a comparative histological and immunophenotypic study with primary sclerosing cholangitis on liver biopsy material. Mod Pathol 22: 1287-1295.

10. Divatia M, Kim SA, Ro JY (2012) IgG4-related sclerosing disease, an emerging entity: a review of a multi-system disease. Yonsei Med J 53: 15-34.

11. Kamisawa T, Okamoto A (2008) IgG4-related sclerosing disease. World J Gastroenterol 14: 3948-3955.

12. Mendes FD, Jorgensen R, Keach J, et al. (2006) Elevated serum IgG4 concentration in patients with primary sclerosing cholangitis. Am J Gastroenterol 101: 2070-2075.

13. Zhang L, Lewis JT, Abraham SC, et al. (2010) lgG4+ plasma cell infiltrates in liver explants with primary sclerosing cholangitis. Am J Surg Pathol 34: 88-94.

14. Batts KP, Ludwig J (1995) Chronic hepatitis. An update on terminology and reporting. Am J Surg Pathol 19: 1409-1417.

15. Ishak K, Baptista A, Bianchi L, et al. (1995) Histological grading and staging of chronic hepatitis. J Hepatol 22: 696-699.

16. Ludwig J, Dickson ER, McDonald GS (1978) Staging of chronic nonsuppurative destructive cholangitis (syndrome of primary biliary cirrhosis). Virchows Arch A Pathol Anat Histol 379: 103-112.

17. Gossard AA, Angulo P, Lindor KD (2005) Secondary sclerosing cholangitis: a comparison to primary sclerosing cholangitis. Am J Gastroenterol 100: 1330-1333.

18. Ghazale A, Chari ST, Zhang L, et al. (2008) Immunoglobulin G4-associated cholangitis: clinical profile and response to therapy. Gastroenterology 134: 706-715.

19. Lee YM, Kaplan MM (1995) Primary sclerosing cholangitis. N Engl J Med 332: 924-933.

20. Nizar AH, Toubi E (2015) IgG4-related disease: Case report and literature review. Auto Immun Highlights 6: 7-15. 